\title{
Comparison of effects of angiotensin II receptor blocker on morning home blood pressure and cardiorenal protection between morning administration and evening administration in hypertensive patients: the COMPATIBLE study
}

\author{
Hisao Mori ${ }^{1}$, Hareaki Yamamoto ${ }^{1}$, Hiroshi Ukai $^{1}$, Shouhei Yuasa ${ }^{1}$, Kazumi Nakajima ${ }^{1}$, Takehiko Mikawa ${ }^{1}$, \\ Masamichi Niizuma ${ }^{1}$, Kouichi Hirao ${ }^{1}$ and Satoshi Umemura ${ }^{2}$; on behalf of the COMPATIBLE Study Group
}

Whether the time of administering the angiotensin receptor antagonist olmesartan influences antihypertensive and renoprotective effectiveness remains unclear. This study compared the effects of olmesartan on morning home blood pressure (MHBP), office BP (OBP) and renoprotective parameters between morning and evening administration. A total of 218 patients with primary hypertension were randomly assigned to receive olmesartan once daily in the morning (morning-dose group) or evening (evening-dose group), and 188 completed the study protocol (morning-dose group, $n=95$; evening-dose group, $n=93$ ). In both groups, morning home systolic BP, morning home diastolic BP, office systolic BP and office diastolic BP decreased significantly. There was no significant difference between the groups in MHBP or OBP after 6 months of treatment. The urinary albumin-to-creatinine ratio (UACR) decreased from 13.9 to $6.9 \mathrm{mg} \mathrm{g}^{-1}$ (geometric means, $P<0.001$ ) in the morning-dose group and from 14.4 to $9.1 \mathrm{mgg}^{-1}(P<0.001)$ in the evening-dose group. The changes in UACR after treatment did not differ significantly between the groups. $\mathrm{SV}_{1}+\mathrm{RV}_{5}$ decreased significantly from baseline to 6 months in the morning-dose group $(P<0.001)$ and the evening-dose group $(P<0.01)$, and did not differ significantly between the groups. In conclusion, olmesartan effectively decreased MHBP, OBP, $\mathrm{SV}_{1}+\mathrm{RV}_{5}$ and UACR regardless of whether the drug was administered in the morning or in the evening. Our results suggest that olmesartan can be prescribed once daily, either in the morning or in the evening.

Hypertension Research (2013) 36, 202-207; doi:10.1038/hr.2012.142; published online 27 September 2012

Keywords: administration time; cardiorenal protection; morning home blood pressure; olmesartan

\section{INTRODUCTION}

A recent study showed that bedtime administration of one or more antihypertensive medications results in better blood pressure (BP) control and greater cardiovascular risk reduction than morning administration. ${ }^{1}$ Although this was an important study based on hard end points, the results have yet to be confirmed. Alternatively, the benefits of antihypertensive therapy can be evaluated on the basis of intermediate surrogate end points, such as microalbuminuria and left ventricular hypertrophy. Several studies ${ }^{2,3}$ have shown that bedtime administration of antihypertensive agents is associated with a greater reduction in urinary albumin-to-creatinine ratio (UACR) than morning administration. However, it remains unclear whether reduced $\mathrm{BP}$ during sleep or the time of drug administration is more important.

Several types of antihypertensive medications, including angiotensin receptor blockers (ARBs) antagonists, ${ }^{4,5}$ have been reported to be free of administration-time-dependent effects on the circadian pattern of BP. To confirm these results, we compared the effects of the ARB olmesartan on morning home BP (MHBP) and cardiovascular and renoprotective parameters between morning and evening administration. Olmesartan was used because it has a terminal halflife of $12-18 \mathrm{~h}^{6}$ and a steady-state elimination half-life of $10.6-16.5 \mathrm{~h}^{7}$ In addition, a recent study showed that the amplitude of the 24-h BP pattern was unaffected by the administration time of olmesartan;

${ }^{1}$ Department of Clinical Research of Kanagawa Association of Medical and Dental Practitioners, Yokohama, Japan and ${ }^{2}$ Department of Medical Science and Cardiorenal Medicine, Yokohama City University Graduate School of Medicine and School of Medicine, Yokohama, Japan

Correspondence: Dr H Mori, Department of Clinical Research of Kanagawa Association of Medical and Dental Practitioners, TS plaza Bldg 2F, Tsuruyatyo 2-23-2, Kanagawa ward, Yokohama, Kanagawa 221-0835, Japan.

E-mail: hmori@gem.hi-ho.ne.jp

Received 24 January 2012; revised 2 August 2012; accepted 9 August 2012; published online 27 September 2012 
however, this study did not evaluate surrogate markers of cardioprotective or renoprotective effectiveness. ${ }^{5}$ In the present study, we tested the hypothesis that the antihypertensive, cardioprotective and renoprotective effects of olmesartan are similar for morning administration and evening administration.

\section{METHODS}

\section{Participants}

Eligible patients had previously untreated primary hypertension, defined as a sitting systolic blood pressure (SBP) of $\geqslant 140 \mathrm{~mm} \mathrm{Hg}$, a diastolic blood pressure (DBP) of $\geqslant 90 \mathrm{~mm} \mathrm{Hg}$, or both, as measured in the office. Age and sex were not restricted. Patients were excluded if they had secondary hypertension, severe liver dysfunction, stroke, bilateral renal artery stenosis, only one kidney, hyperpotassemia or a serum creatinine $(\mathrm{sCr})$ level of $\geqslant 3.0 \mathrm{mg} \mathrm{dl}^{-1}$, of if they regularly used nonsteriodal anti-inflammatory drugs or steroid hormones. Pregnant women were also excluded. The study was performed from 1 July 2005 through 28 February 2010, and the study protocol was registered on a clinical trial registration site UMIN-CTR (University Hospital Medical Information Network-Clinical Trials Registry). ${ }^{8}$ The protocol was approved by the Ethical Review Boards of the Kanagawa Association of Medical and Dental Practitioners, and all participants provided written informed consent. The study was conducted in accordance with the Declaration of Helsinki.

\section{Study design}

We calculated the sample size for this trial as follows: assuming a s.d. of $12 \mathrm{~mm} \mathrm{Hg}$ for morning home SBP, $80 \%$ of the study showed a significant difference of $5 \mathrm{~mm} \mathrm{Hg}$ in the mean of morning home SBP between the morning-dose group and the evening-dose group at the level of $P<0.05$ (two-tailed); the numbers of sample of each group were 91.

Eligible patients were randomly assigned to the morning-dose group or the evening-dose group by means of numbered containers. In both groups, the initial dose of olmesartan was 10-20 mg daily, and was titrated up to $40 \mathrm{mg}$ as required to reach the target $\mathrm{BP}$. Every patient was instructed to visit each clinic every month and if the office BP (OBP) did not reach the target BP even after the dose had been increased to $40 \mathrm{mg}$ of olmesartan, patients additionally received a long-acting calcium channel blocker (CCB) (Figure 1).

Furthermore, OBP did not reach the target BP after additional CCB was administered, then patients received the third medicine (angiotensinconverting enzyme inhibitors, beta-blockers, diuretics or spironolactone).

In the morning-dose group, patients were instructed to take olmesartan and added CCB or the third medicine in the morning on awaking from night-time sleep after breakfast, and in the evening-dose group patients were instructed to take olmesartan and added CCB or the third medicine in the evening before going to bed. OBP measurements were obtained after the patient had rested in a seated position for $\geqslant 10 \mathrm{~min}$, using validated automatic oscillometric device or mercury BP manometer. MHBP measurements were obtained in a sitting position after $2 \mathrm{~min}$ of rest in the morning within $1 \mathrm{~h}$ after awaking from night-time sleep, after micturition, before ingestion of drugs, before breakfast. MHBP measurements were performed everyday throughout the trial, using automatic oscillometric device (HEM7000; Omron Healthcare Kyoto, Japan or ES-P302; Terumo, Tokyo, Japan). Patients were asked to record their MHBP on the recording charts. The target BP was defined as an office systolic BP (OSBP)

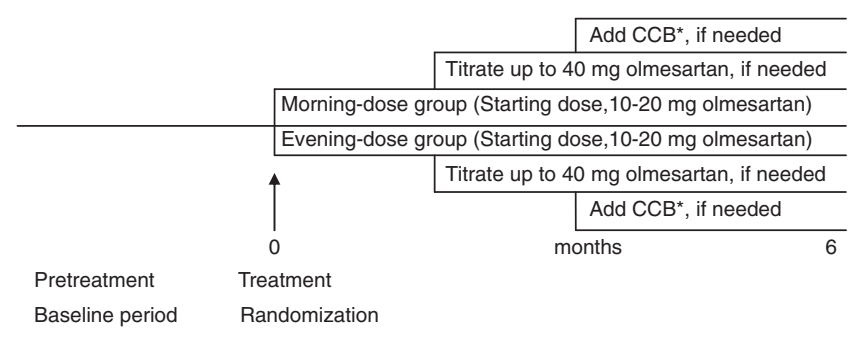

Abbreviations: ${ }^{*} \mathrm{CCB}$, long-acting calcium channel blocker.

Figure 1 Study design. of $<140 \mathrm{~mm} \mathrm{Hg}$ and an office diastolic BP (ODBP) of $<90 \mathrm{~mm} \mathrm{Hg}$ in patients 65 years or older, and an OSBP of $<130 \mathrm{~mm} \mathrm{Hg}$ and an ODBP of $<85 \mathrm{~mm} \mathrm{Hg}$ in patients younger than 65 years. In patients who had diabetes mellitus or chronic kidney disease, the target OSBP was $<130 \mathrm{~mm} \mathrm{Hg}$, and the target ODBP was $<80 \mathrm{~mm} \mathrm{Hg}$.

\section{Evaluation of renal function}

Renal function was evaluated on the basis of the estimated glomerular filtration rate (eGFR), which was calculated using an equation developed for the Japanese population, as recommended by the Japanese Society of Nephrology. ${ }^{9}$

$$
\mathrm{eGFR}=194 \times \mathrm{sCrn}^{-1.094} \times \mathrm{age}^{-0.287}(\times 0.739, \text { if female }) .
$$

\section{Statistical analysis}

Baseline characteristics other than sex, administration rate of diabetic medicines and percentage of chronic kidney disease (eGFR $<60 \mathrm{ml} \mathrm{min}^{-1}$ $1.73 \mathrm{~m}^{-2}$ ) were analyzed with the use of non-paired $t$-tests. Fisher's exact method was used to analyze sex, administration rate of diabetic medicines and percentage of chronic kidney disease. Changes in OBP, office pulse rate, morning home pulse rate, cardiothoracic ratio (CTR; divided transverse diameter of the heart was measured as the sum of the widest portions of the heart to the right and to the left of the midline of the spine by the internal diameter of the chest measured at the level of the highest point on the right hemidiaphragm), $S V_{1}+R V_{5}$ (total voltage of the $S$ wave of $V_{1}+R$ wave of $\mathrm{V}_{5}$ ), eGFR and $\mathrm{s} C r$ in each group were analyzed with paired $t$-tests. Non-paired $t$-tests were used to compare OBP, office pulse rate, MHBP and morning home pulse rate between the morning-dose group and the evening-dose group, and $\chi^{2}$-tests were used to compare antihypertensive regimens between the groups. The significance of differences in UACR and high-sensitivity C-reactive protein (hsCRP) were analyzed with the Wilcoxon signed-rank test and Mann-Whitney $U$ test, because these variables did not show a Gaussian distribution even after conversion to common logarithms. Analyses were carried out with SPSS version 11 software (SPSS Chicago, IL, USA). All values are expressed as means \pm s.d., and $P$-values of $<0.05$ were considered to indicate statistical significance.

\section{RESULTS}

\section{Patient characteristics}

A total of 218 patients were enrolled and randomly assigned to the morning-dose group or the evening-dose group. Of the 110 patients assigned to the morning-dose group, 15 were excluded because of loss to follow-up $(n=11)$ or death of their physician $(n=4)$. Of the 108 patients assigned to the evening-dose group, 15 were excluded because of loss to follow-up $(n=13)$ or death of their physician $(n=2)$.

Table 1 shows the characteristics of the 188 eligible subjects randomly assigned to the morning-dose group $(n=95)$ or the evening-dose group $(n=93)$. Because of mis-collection of biochemical data, some differences in this data were found. There were no significant differences between the two groups in baseline characteristics such as age, gender, anthropometric data, low-density lipoprotein cholesterol, high-density lipoprotein cholesterol, triglycerides, sCr, sodium, potassium, chloride, fasting plasma glucose, HbA1c, administration rate of diabetic medicines or percentage of chronic kidney disease.

\section{Changes in blood pressure}

After 6 months of treatment, OSBP, ODBP, MHSBP, and MHDBP were significantly lower than the respective baseline values in both the morning-dose group and the evening-dose group. Office pulse rate and morning home pulse rate significantly decreased after 6 months of treatment in the morning-dose group, but not in the evening-dose group. At baseline and 6 months of treatment, OSBP, ODBP, office pulse rate, MHSBP, MHDBP, morning home pulse rate, $\mathrm{SV}_{1}+\mathrm{RV}_{5}$, 
Table 1 Baseline characteristics of morning-dose group and eveningdose group

\begin{tabular}{|c|c|c|c|}
\hline & $\begin{array}{l}\text { Morning-dose } \\
\text { group }\end{array}$ & $\begin{array}{c}\text { Evening-dose } \\
\text { group }\end{array}$ & P-value \\
\hline Male & 57 & 49 & \\
\hline Female & 38 & 44 & $0.194^{a}$ \\
\hline Age (years) & $\begin{array}{c}62.1 \pm 13.6 \\
(n=95)\end{array}$ & $\begin{array}{c}60.8 \pm 9.4 \\
(n=93)\end{array}$ & $0.451^{\mathrm{b}}$ \\
\hline Height $(\mathrm{cm})$ & $\begin{array}{c}161.1 \pm 10.6 \\
(n=94)\end{array}$ & $\begin{array}{c}161.6 \pm 10.4 \\
(n=93)\end{array}$ & $0.795^{b}$ \\
\hline Weight (kg) & $\begin{array}{c}63.7 \pm 13.7 \\
(n=95)\end{array}$ & $\begin{array}{c}63.8 \pm 14.4 \\
(n=93)\end{array}$ & $0.951^{\mathrm{b}}$ \\
\hline Abdominal circumference $(\mathrm{cm})$ & $\begin{array}{c}84.6 \pm 9.0 \\
(n=94)\end{array}$ & $\begin{array}{c}83.9 \pm 10.7 \\
(n=89)\end{array}$ & $0.611^{b}$ \\
\hline BMI $\left(\mathrm{kg} \mathrm{m}^{-2}\right)$ & $\begin{array}{c}24.4 \pm 3.6 \\
(n=94)\end{array}$ & $\begin{array}{c}24.3 \pm 3.9 \\
(n=93)\end{array}$ & $0.834^{b}$ \\
\hline LDL-cholesterol (mg dl-1) & $\begin{array}{c}127.8 \pm 28.6 \\
(n=90)\end{array}$ & $\begin{array}{c}124.8 \pm 35.3 \\
(n=90)\end{array}$ & $0.520^{\mathrm{b}}$ \\
\hline HDL-cholesterol (mg dl-1) & $\begin{array}{c}63.0 \pm 16.6 \\
(n=94)\end{array}$ & $\begin{array}{c}62.1 \pm 14.6 \\
(n=93)\end{array}$ & $0.694^{\mathrm{b}}$ \\
\hline Triglycerides $\left(\mathrm{mg} \mathrm{dl}^{-1}\right)$ & $\begin{array}{c}156.4 \pm 107.9 \\
(n=95)\end{array}$ & $\begin{array}{c}136.0 \pm 86.3 \\
(n=93)\end{array}$ & $0.156^{\mathrm{b}}$ \\
\hline Creatinine $\left(\mathrm{mg} \mathrm{dl}^{-1}\right)$ & $\begin{array}{c}0.79 \pm 0.55 \\
(n=95)\end{array}$ & $\begin{array}{c}0.71 \pm 0.17 \\
(n=93)\end{array}$ & $0.204^{b}$ \\
\hline $\mathrm{Na}$ (mequiv. $1^{-1}$ ) & $\begin{array}{c}141.5 \pm 2.0 \\
(n=94)\end{array}$ & $\begin{array}{c}141.2 \pm 2.0 \\
(n=92)\end{array}$ & $0.366^{\mathrm{b}}$ \\
\hline $\mathrm{K}$ (mequiv. $\mathrm{I}^{-1}$ ) & $\begin{array}{c}4.3 \pm 0.7 \\
(n=94)\end{array}$ & $\begin{array}{c}4.2 \pm 0.5 \\
(n=93)\end{array}$ & $0.379^{b}$ \\
\hline $\mathrm{Cl}$ (mequiv. $\mathrm{I}^{-1}$ ) & $\begin{array}{c}103.4 \pm 2.3 \\
(n=93)\end{array}$ & $\begin{array}{c}103.4 \pm 2.6 \\
(n=93)\end{array}$ & $0.953^{b}$ \\
\hline Fasting plasma glucose $\left(\mathrm{mg} \mathrm{dl}^{-1}\right)$ & $\begin{array}{c}107.2 \pm 23.7 \\
(n=92)\end{array}$ & $\begin{array}{c}108.9 \pm 42.3 \\
(n=92)\end{array}$ & $0.733^{b}$ \\
\hline $\mathrm{HbA} 1 \mathrm{c}(\%)$ & $\begin{array}{l}5.3 \pm 0.6 \\
(n=93)\end{array}$ & $\begin{array}{l}5.5 \pm 1.1 \\
(n=90)\end{array}$ & $0.196^{b}$ \\
\hline Administration of diabetic medicines & $9.5 \%(n=95)$ & $8.6 \%(n=93)$ & $0.519^{a}$ \\
\hline Percentage of CKD & $6.3 \%(n=95)$ & $6.5 \%(n=93)$ & $0.602^{a}$ \\
\hline
\end{tabular}

Abbreviations: BMI, body mass index; CKD, chronic kidney disease; HDL, high-density lipoprotein; LDL, low-density lipoprotein.

aFisher's exact method.

bnon-paired t-test.

CTR, sCr, eGFR, hsCRP and UACR showed no significant difference between morning-dose group and evening-dose group (Table 2); Figure 2 shows the time courses of OBP and MHBP during the study. There was no significant difference at any month between the morning-dose group and the evening-dose group. There were also no significant differences between the groups in the reductions in BPs or PRs from baseline to 6 months after treatment began (Table 3).

Changes in $\mathrm{SV}_{1}+\mathrm{RV}_{5}$, CTR, creatinine, eGFR, UACR and hsCRP Table 2 shows the changes in $\mathrm{SV}_{1}+\mathrm{RV}_{5}$, CTR, sCr, eGFR, UACR and hsCRP from baseline to after 6 months of treatment. In the morningdose group, $\mathrm{SV}_{1}+\mathrm{RV}_{5}$, eGFR and UACR decreased significantly, but $\mathrm{CTR}, \mathrm{SCr}$ and hsCRP showed no significant change. In the eveningdose group, $\mathrm{SV}_{1}+\mathrm{RV}_{5}$, CTR and UACR decreased significantly, with no significant change in $\mathrm{sCr}$, eGFR or hsCRP. Figure 3 compares the changes in sCr, eGFR, $\mathrm{SV}_{1}+\mathrm{RV}_{5}$, CTR, UACR and hsCRP from baseline to after 6-months' treatment between the morning-dose group and the evening-dose group. The magnitudes of the changes in these variables did not differ significantly between the groups.
Doses of olmesartan and combination regimens

The starting doses of olmesartan were $14.0 \pm 4.9 \mathrm{mg}(n=96)$ in the morning-dose group and $14.0 \pm 4.9 \mathrm{mg}(n=93)$ in the evening-dose group, and the doses at 6 months of treatment were $22.0 \pm 10.6 \mathrm{mg}$ $(n=96)$ in the morning-dose group and $21.2 \pm 11.6 \mathrm{mg}(n=93)$ in the evening-dose group. Throughout this trial, there was no significant difference in the dose of olmesartan between the groups (non-paired $t$-test).

The mean numbers of antihypertensive regimens were 1.14 in the morning-dose group and 1.16 in the evening-dose group at baseline, and 1.40 in the morning-dose group and 1.42 in the evening-dose group at 6 months of treatment. Concurrently, the prescribed drugs included angiotensin-converting enzyme inhibitors, beta-blockers, CCBs, diuretics and spironolactone. There was no significant difference in concurrently prescribed combination regimens between the groups during any part of the trial $\left(\chi^{2}\right.$-test).

\section{DISCUSSION}

Our study showed that olmesartan significantly reduced OBP and MHBP from baseline to the end of 6 months of treatment in both the morning-dose and evening-dose groups. There was no significant difference in the baseline OSBP between morning-dose group and evening-dose group, but the $P$-value was 0.052. Considering the statistical significance, OSBP lowering effect of olmesartan between morning-dose group and evening dose-group may be necessary to confirm again. In addition, $\mathrm{SV}_{1}+\mathrm{RV}_{5}$ and UACR significantly decreased in both the morning-dose and evening-dose groups, and the changes in these variables from baseline did not differ significantly between the groups. CTR significantly decreased in the evening-dose group, and eGFR significantly decreased in the morning-dose group.

Bedtime administration of ramipril was shown to be significantly more effective than morning administration for reducing nocturnal BP. ${ }^{10}$ In contrast, valsartan effectively reduced BP throughout a $24-\mathrm{h}$ period, irrespective of whether the drug was administered in the morning or at bedtime. ${ }^{4}$ Another study reported that bedtime treatment with telmisartan more effectively controlled nocturnal BP than morning treatment, despite the prolonged half-life of this drug. ${ }^{11}$ Evening administration of guanabenz or clonidine has also been shown to significantly lower morning BP. ${ }^{12}$ These findings suggest that bedtime administration of ARBs, angiotensin-converting enzyme inhibitors and central $\alpha_{2}$-agonists is therapeutically beneficial because it lowers morning BP more effectively than does morning administration. Effective lowering of morning BP has an important role in therapy, because morning hypertension is the strongest independent predictor of a future clinical stroke. ${ }^{13}$ Recently, the MAPEC study showed that bedtime administration of antihypertensive regimens improved BP control, decreased the prevalence of a non-dipper pattern (that is, no nocturnal reduction in BP), and significantly reduced morbidity and mortality owing to cardiovascular disease. ${ }^{1,14}$ Bedtime administration of candesartan ${ }^{2}$ and valsartan ${ }^{3}$ reduced UACR to significantly greater extents than morning administration, suggesting that bedtime administration of ARBs more effectively reduces UCAR and morning BP than does morning administration. In our study, both morning administration and evening administration of olmesartan significantly reduced MHBP and UACR to similar extents. It is unclear why olmesartan reduced MHBP and UACR regardless of the administration time in our study. However, the prolonged duration of action of olmesartan demonstrated in previous studies ${ }^{6,7,8}$ might have contributed to equivalent lowering of BP and UACR irrespective of administration time. These results are consistent with the findings of a previous study in which both morning 
Table 2 Changes in OBP, OPR, MHBP, MHPR, SV1 + RV5, CTR, creatine, eGFR, hsCRP and UACR from baseline to after 6 months of treatment, and comparison between morning-dose and evening-dose groups

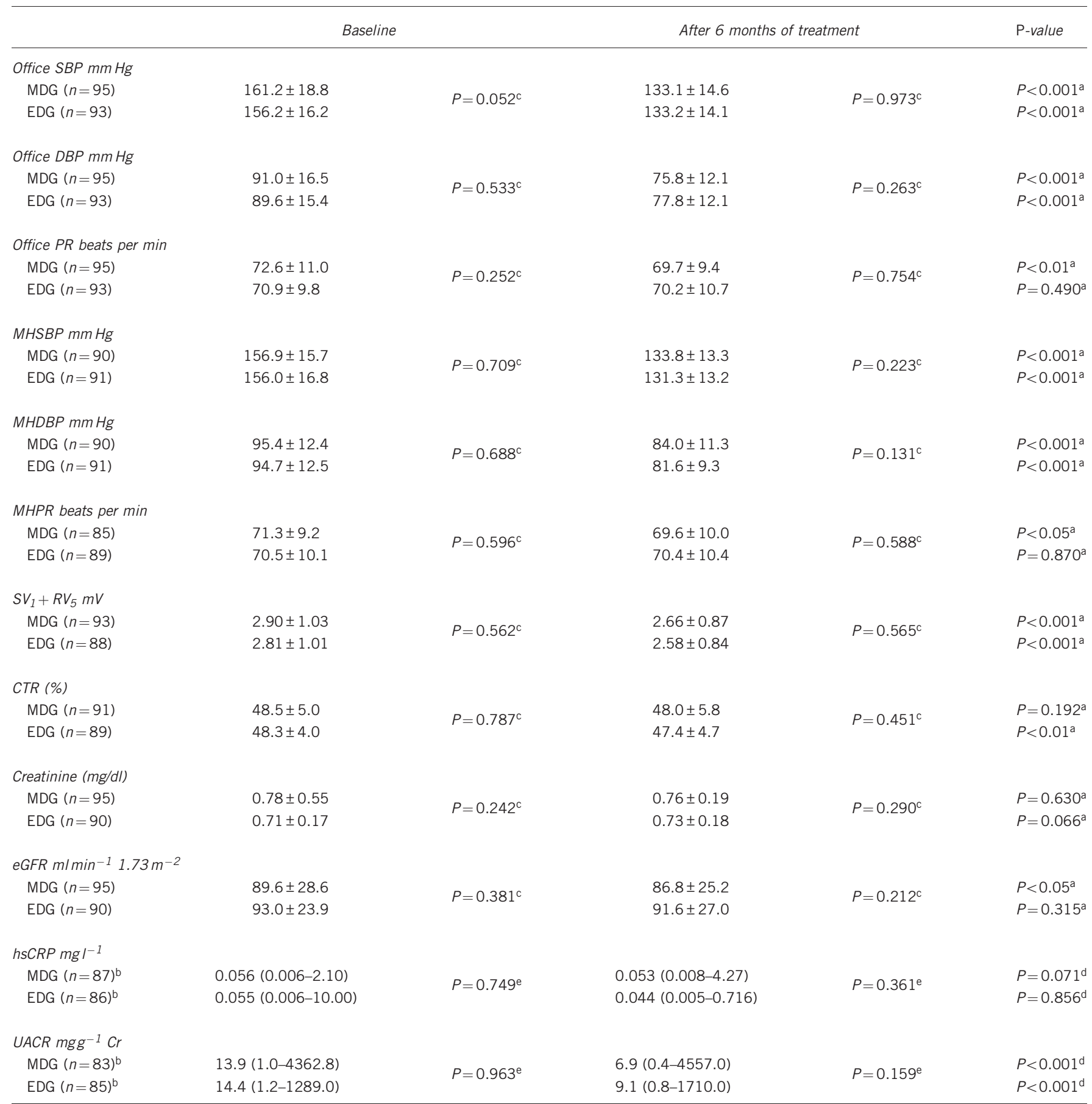

Abbreviations: CTR, cardiothoracic ratio; DBP, diastolic blood pressure; EDG, evening-dose group; eGFR, estimated glomerular filtration rate; hsCRP, high-sensitivity C-reactive protein; MDG, morning-dose group; MHDBP, morning home diastolic blood pressure; MHPR, morning home pulse rate; MHSBP, morning home systolic blood pressure; PR, pulse rate; UACR, urinary albumin-to-

creatinine ratio.
apaired $t$-test.

bValues are geometric means, with ranges in parentheses.

cnon-paired $t$-test.

dWilcoxon signed-rank test.

eMann-Whitney $U$ test.

administration and evening administration of olmesartan similarly reduced $\mathrm{BP}$, but the test for UACR was lacking. ${ }^{5}$ In the present study, we concluded olmesartan decreased UACR equivalently, regardless of whether the drug was administered in the morning or at bedtime, but the limitation of interpretation may be considered because UACR samples were collected at different times according to the patients' usual visiting the offices. Bedtime administration of candesartan ${ }^{2}$ and valsartan ${ }^{3}$ reduced UACR effectively than does 

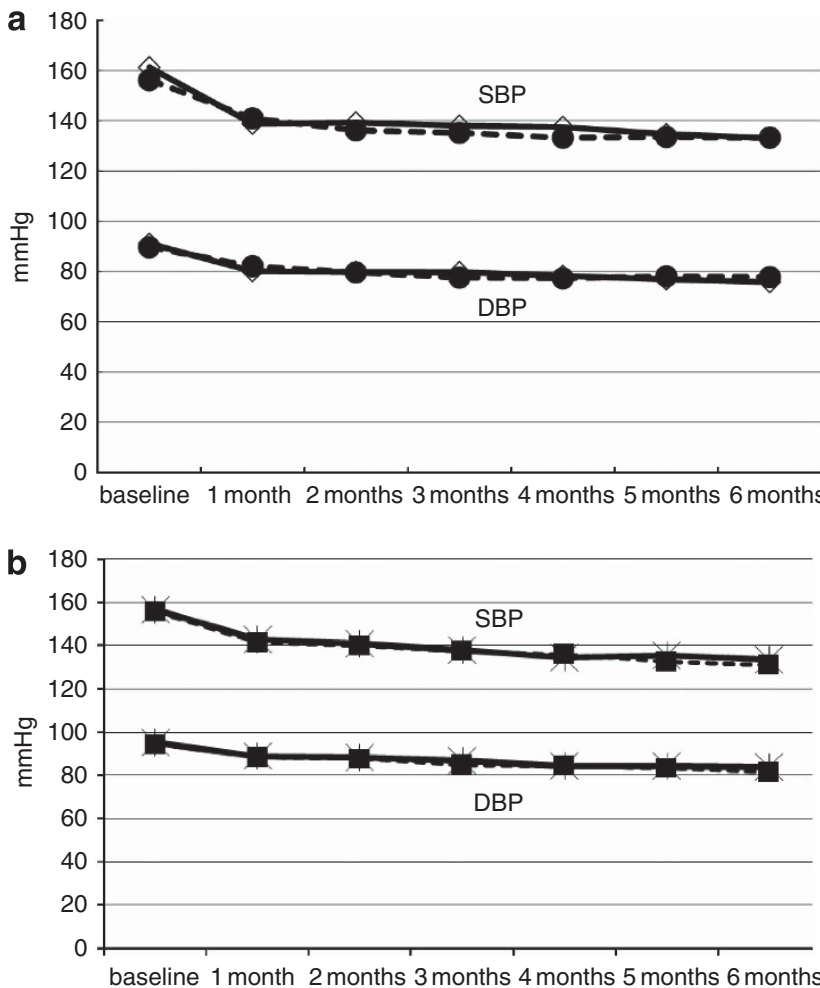

Figure 2 (a) Time courses of office mean blood pressures from baseline to after 6 months of treatment in morning-dose group $(\diamond, n=95)$ and evening-dose group ( $\bullet n=93$ ). (b) Time course of morning home blood pressure from baseline to after 6 months of treatment in morning-dose group ( $\left.{ }^{*}, n=90\right)$ and evening-dose group ( $\left.\mathbf{\square}, n=91\right)$.

Table 3 Changes in BP and pulse rate from baseline to after 6 months of treatment

\begin{tabular}{llll}
\hline & Morning-dose group & Evening-dose group & $P$-value \\
\hline Office & & & \\
SBP & & & \\
$\quad$ Baseline (mm Hg) & $161.2 \pm 18.8(n=95)$ & $156.2 \pm 16.2(n=93)$ & 0.052 \\
$\quad$ Change $(\mathrm{mm} \mathrm{Hg})$ & $-28.1 \pm 18.0$ & $-23.0 \pm 17.4$ & 0.050 \\
DBP & & & \\
$\quad$ Baseline (mm Hg) & $91.0 \pm 16.5(n=95)$ & $89.6 \pm 15.4(n=93)$ & 0.533 \\
Change (mm Hg) & $-15.2 \pm 11.6$ & $-11.7 \pm 14.4$ & 0.073 \\
PR & & & \\
Baseline (bpm) & $72.6 \pm 11.0(n=95)$ & $70.9 \pm 9.8(n=93)$ & 0.252 \\
Change (bpm) & $-2.9 \pm 9.5$ & $-0.7 \pm 9.4$ & 0.112 \\
Home & & & \\
MHSBP & & & \\
Baseline (mm Hg) & $156.9 \pm 15.7(n=90)$ & $156.0 \pm 16.8(n=91)$ & 0.438 \\
Change (mm Hg) & $-23.2 \pm 15.9$ & $-24.7 \pm 16.5$ & 0.768 \\
MHDBP & & & \\
Baseline (mm Hg) & $95.4 \pm 12.4(n=90)$ & $94.7 \pm 12.5(n=91)$ & 0.414 \\
Change (mm Hg) & $-11.5 \pm 10.9$ & $-13.0 \pm 10.8$ & 0.325 \\
MHPR & & & \\
Baseline (bpm) & $71.3 \pm 9.2(n=90)$ & $70.5 \pm 10.1(n=89)$ & 0.429 \\
Change (bpm) & $-1.7 \pm 7.4$ & $-0.1 \pm 7.3$ & 0.151 \\
\hline
\end{tabular}

Abbreviations: DBP, diastolic blood pressure; MHDBP, morning home diastolic blood pressure; MHPR, morning home pulse rate; MHSBP, morning home systolic blood pressure; PR, pulse rate; SBP, systolic blood pressure; bpm, beats per minute.

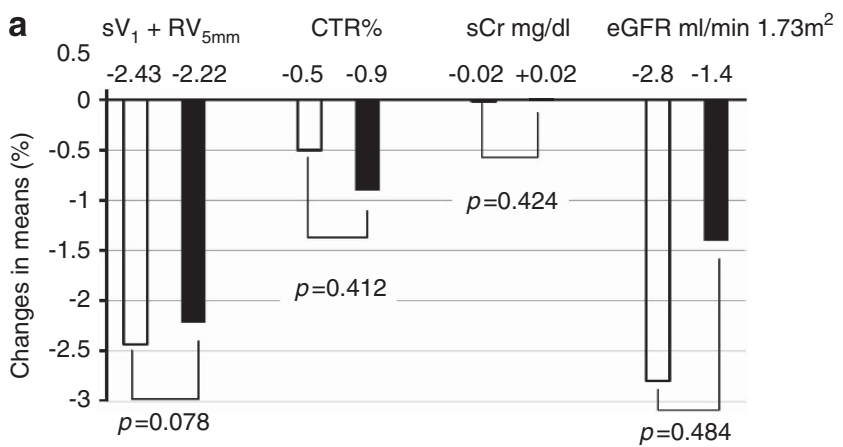

b

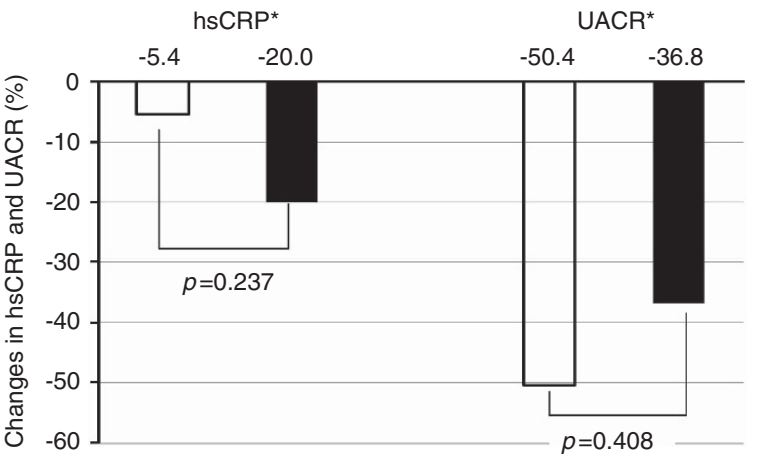

Figure 3 (a) Comparison of changes in $\mathrm{SV}_{1}+\mathrm{R}_{5}, \mathrm{CTR}$, creatinine and eGFR from baseline to after 6 months of treatment between the morning-dose group and evening-dose group (non-paired $t$-test). (b) Comparison of changes in hSCRP and UACR changes from baseline to after 6 months of treatment between the morning-dose group and evening-dose group (Mann-Whitney $U$ test). CTR, cardiothoracic ratio; eGFR, estimated glomerular filtration rate; hsCRP, high-sensitivity C-reactive protein; UACR, urinary albumin-to-creatinine ratio; *, Values are \% changes in geometric means from baseline; $\square$, morning-dose group; $\boldsymbol{\square}$, evening-dose group.

morning administration, but there is little study for other ARBs concerning administration time and UACR. Renoprotective point of view the same studies are expected to be carried out

Previous studies have shown that ARBs exert anti-inflammatory activity by blocking angiotensin $\mathrm{II},{ }^{15,16}$ and increased CRP levels are associated with hypertension. ${ }^{17}$ Because olmesartan significantly decreases hsCRP levels in patients with diabetes mellitus, ${ }^{18,19}$ we monitored hsCRP levels throughout the study. However, no significant change in hsCRP levels was detected. The reason why olmesartan did not significantly alter hsCRP levels in our study is unclear.

We cannot assess the effect of asleep BPs and evening home BP because we did not collect asleep BPs by ambulatory blood pressure monitoring or evening home BP. Consequently, the limitation of interpretation may be considered as to the results of the study. The changes of office and morning home PR significantly decreased in the morning-dose group but there was no significant change found in evening-dose group. The reason why PR decreased in the morningdose group is not clear. We consider the need to confirm the difference of PR change according to the time of administration of olmesartan-based antihypertensive treatment. The CTR decreased significantly in the evening-dose group and eGFR decreased significantly in the morning-dose group. These reasons were not clear, but our study period for every participant was 6 months, and a longer study period may be needed to assess the change of CTR and eGFR.

In conclusion, olmesartan decreased OBP, MHBP, UACR and $\mathrm{SV}_{1}+\mathrm{RV}_{5}$, regardless of whether the drug was administered in the 
morning or at bedtime. Our results suggest that olmesartan can be prescribed once daily, either in the morning or evening.

\section{CONFLICT OF INTEREST}

The authors declare no conflict of interest.

\section{ACKNOWLEDGEMENTS}

This study was supported by a research grant from Kanagawa Association of Medical and Dental Practitioners. The study was designed, conducted and interpreted by comparison of effects of angiotensin II receptor blocker on MHBP and cardiorenal protection between morning administration and evening administration in hypertensive patients: the COMPATIBLE study group belong to the Department of Clinical Research of Kanagawa Association of Medical and Dental Practitioners. Members of COMPATIBLE study group: Principal investigator: Hisao Mori, Cooperative researchers; Hareaki Yamamoto, Hiroshi Ukai, Shouhei Yuasa, Kazumi Nakajima, Takehiko Mikawa, Masamichi Niizuma, Kouichi Hirao, Interpretation advisor; Satoshi Umemura.

1 Hermida RC, Ayala DE, Mojón A, Fernández JR. Influence of circadian time of hypertension treatment on cardiovascular risk: result of the MAPEC study. Chronobiol Int 2010; 27: 1629-1651.

2 Kario K, Hoshide S, Shimizu M, Yano Y, Eguchi K, Ishikawa J, Shimada K. Effect of dosing time of angiotensin II receptor blockade titrated by self-measured blood pressure recordings on cardiorenal protection in hypertensives: the Japan morning surge-target organ protection (J-TOP) study. J Hypertens 2010; 28: 1574-1583.

3 Hermida RC, Calvo C, Ayala DE, López JE. Decrease in urinary albumin excretion associated with the normalization of nocturnal blood pressure in hypertensive subjects. Hypertension 2005; 46(part 2): 960-968.

4 Hermida RC, Calvo C, Ayala DE, Domínguez MJ, Covelo M, Fernández JR, Mojón A, López JE. Administration time-dependent effects of valsartan on ambulatory blood pressure in hypertensive subjects. Hypertension 2003; 42: 283-290.

5 Smolensky MH, Hermida RC, Portaluppi F. Comparison of the efficacy of morning versus evening administration of olmesartan in uncomplicated essential hypertension. Chromobiol Int 2007; 24: 171-181.
6 Schwocho LR, Masonson HN. Pharmaocokinetics of CS-866, a new angiotensin II receptor blocker, in healthy subjects. J clin Pharmacol 2001; 41: 515-527.

7 von Bergmann K, Laeis P, Puchler K, Sudhop T, Schwocho LP, Gonzalez L. Olmesartan medoxomil: influence of age, renal and heptic function on the pharmacokinetics of olmesartan medoxomil. J Hypertens Supp/ 2001; 19(Suppl 1): S33-S40.

8 Mori H. UMIN000003238. Available at https://upload.umin.ac.jp/cgi-open-bin/ctr/ ctr.cgi.

9 Matsuo S, Imai E, Horio M, Yasuda Y, Tomita K, Nitta K, Yamagata K, Yomino Y, Yokoyama $\mathrm{H}$, Hishida A. Collaborators developing the Japanese equation for estimated GFR. Revised equations for estimated GFR from serum creatinine in Japan. Am J Kidney Dis 2009; 53: 982-992.

10 Hermida RC, Ayala DE. Chronotherapy with the angiotensin-converting enzyme inhibitor ramipril in essential hypertension: improved blood pressure control with bedtime dosing. Hypertension 2009; 54: 40-46.

11 Hermida RC, Ayala DE, Fernández JR, Calvo C. Comparison of the efficacy of morning versus eveming administration of telmisartan in essential hypertension. Hypertension 2007; 50: 715-722.

12 Hashimoto J, Chonan K, Aokib Y, Ugajin T, Yamaguchi J, Nishimura T, Kikuya M, Michimata M, Matsubara M, Araki T, Ohkubo T, Imai Y. Therapeutic effects of evening administration of guanabenz and clonidine on morning hypertension: evaluation using home-based blood pressure measurements. J Hypertens 2003; 21: 805-811.

13 Kario K, Ishikawa J, Pickering TG, Hoshide S, Eguchi K, Morinari M, Hoshide Y, Kuroda T, Shimada K. Morning hypertension: the strongest independent risk factor for stroke in elderly hypertensive patients. Hypertens Res 2006; 29: 581-587.

14 Portaluppi F, Smolensky MH. Perspectives on the chronotherapy of hypertension based on the results of the MAPEC study. Chronobiol Int 2010; 27: 1652-1667.

15 Dzau VJ. Theodore Cooper lecture: tissue angiotensin and pathobiology of vascular disease: a unifying hypothesis. Hypertension 2001; 37: 1047-1052.

16 Ferrario $C$. Effect of angiotensin receptor blockade on endotherial function: focus on olmesartan medoxomil. Vasc Health Risk Manag 2009; 5: 301-314.

17 Tan X, Zhong J, Weijun T, Wei H, Yanbin L, Li Z, Yonghong Z. Relationship of $\mathrm{C}$-reactive protein with hypertension and interactions between increased C-reactive protein and other risk factors on hypertension in Mongolian people, China. Circ J 2008; 72: $1324-1328$.

18 de Vinuesa SG, Goicoechea M, Kanter J, Puerta M, Cachofeiro V, Lahera V, GómezCampdeá $\mathrm{F}$, Luńo J. Insulin resistance, inflammatory biomarkers, and adipokines in patients with chronic kidney disease: effect of angiotensin II blockade. Am Soc Nephrol 2006; 17(12 suppl 3): S206-S212.

19 Nakayama S, Watada H, Mita T, Ikeda F, Shimizu T, Uchino H, Fujitani Y, Hirose T, Kawamori R. Comparison of effects of olmesartan on blood pressure and metabolic parameters in Japanese early-stage type-2 diabetics with hypertension. Hypertens Res 2008; 31: 7-13. 